\title{
Evaluación de la respuesta terapéutica en pacientes con enfermedad artrósica por farmacéuticos comunitarios
}

\author{
Alicia González Rodríguez, Concepción Ledo Olivares, Rosario Marina Llorente Pérez, \\ Mercedes Piñeiro Arias, Rafael Navarro Alayeto \\ Licenciados en Farmacia. Farmacéuticos comunitarios en Madrid.
}

\section{PALABRAS CLAVE \\ osteoartrosis, evaluación de la respuesta terapéutica, respuesta clínica mínima efectiva, problemas relacionados con el medicamento, resultados negativos de la medicación, farmacia comunitaria, atención farmacéutica}

\section{ABREVIATURAS}

OA: osteoartrosis. EVA: escala visual analógica. WOMAC: cuestionario de calidad de vida Western Ontario McMaster University Osteoarthritis Index.

SMOADS: Symptom Modifying Osteoarthritis Drugs.

SYSADOA: Symptomatic Slow Acting Drugs for Osteoarthritis. SER: Sociedad Española de Reumatología.

EULAR: European League Against Rheumatism o Liga Europea contra el Reumatismo. ACR: American College of Rheumatology.

OARSI: Osteoarthritis Research Society International. PRM: problemas relacionados con el medicamento.

RNM: resultados negativos de la medicación.

IPM: información personalizada del medicamento.

IF: indicación farmacéutica.

SFT: seguimiento

farmacoterapéutico.

EPS: Educación para la Salud. PS: problema de salud.

FC: farmacia comunitaria. MAP: médico de atención primaria.

AINE: antiinflamatorios no esteroideos.

COXIB: inhibidores selectivos de la ciclooxigenasa.

\section{RESUMEN}

Introducción: La artrosis es una enfermedad que origina dolor, rigidez e incapacidad funcional. Actualmente el tratamiento es sintomático, no existen fármacos con capacidad para frenar la enfermedad artrósica, aunque los síntomas que origina se pueden aliviar, permitiendo que el paciente padezca lo menos posible.

Objetivos: Evaluar la respuesta terapéutica en pacientes con osteoartrosis desde la farmacia comunitaria.

Material y métodos: Estudio cuasi experimental no aleatorizado realizado en farmacias comunitarias de Madrid. Se cumplimentó un cuestionario estructurado recogiendo información sobre el paciente, diagnóstico, medicamentos prescritos, articulaciones afectadas, grado de dolor, estado funcional de la articulación, estado global del paciente e intervención farmacéutica realizada.

Resultados: La muestra fue constituida por 141pacientes. Los fármacos incluidos pertenecieron a los grupos N02B (36,1\%), M01A (27,6\%), N02AX (23,4\%), M01AH (14,1\%), M01AX $(27,6 \%)$ prescritos para osteoartrosis. Con relación al dolor, $34(24,1 \%)$ pacientes obtuvieron una reducción del dolor de $20 \mathrm{~mm}$ en la EVA, alcanzando la respuesta clínica mínima efectiva. Respecto a la calidad de vida, la actividad más afectada fue la de subir y bajar escaleras, presentado muchísimo dolor al subir y bajar escaleras 58 (41,1\%) sujetos. Detectamos 111 RNM, de los que 61 fueron de inefectividad (54,9\%). La intervención farmacéutica fue ofrecer educación sanitaria en el autocuidado de las articulaciones a 29 (20,5\%) pacientes, recomendar ejercicio físico a 32 (22,7\%), recomendar dieta-ejercicio a 23 (16,3\%), derivar al especialista a $19(13,4 \%)$, derivar al MAP a $12(8,5 \%)$.

Conclusión: La intervención del farmacéutico comunitario en pacientes con osteoartrosis contribuye a mejorar la efectividad de los tratamientos, mejorando los resultados de salud de los mismos.

Evaluation by community pharmacists of therapeutic response in patients with arthrotic disease

\section{ABSTRACT}

Introduction: Osteoarthritis is a disease that causes pain, stiffness and functional disability. Nowadays the treatment is symptomatic, do not exist drug to stop the osteoarthritis disease, allowing patients should suffer as little as possible.

Objectives: To evaluate the therapeutic response in osteoarthritis patients from community pharmacy.

Material \& Methods: Quasi experimental not randomized study realized in community pharmacies from Madrid. A structured questionnaire was completed, gathering information about patients: medical report, prescribed drug, affected joint, degree of pain, functional joint condition, patient general condition and pharmaceutical realized intervention.

Results: The sample was constituted by 141 patients. The included drugs belong to groups: N02B (36.1\%), M01A (27.6\%), N02AX (23.4\%), M01AH (14.1\%), M01AX (27.6\%) prescribed for osteoarthritis. With relation to the pain, $34(24.1 \%)$ patients had a reduction of pain of $20 \mathrm{~mm}$ in VAS (Visual Analogue Scale), reaching clinically significant improvement.

Este trabajo fue presentado en formato póster al V Congreso Nacional de Farmacéuticos Comunitarios, celebrado en Barcelona en noviembre de 2012, y obtuvo el primer premio en la categoría de Dispensación.

Recibido: 3-12-2013

Aceptado: 18-5-2014

Disponible online: 1-6-2014
Financiación: Ninguna ajena.

Conflicto de intereses: Los autores declaran no tener ningún conflicto de intereses en relación con el presente articulo.

Cite este artículo como: González A, Ledo C, Llorente RM, Piñeiro M, Navarro R. Evaluación de la respuesta terapéutica en pacientes con enfermedad artrósica por farmacéuticos comunitarios. Farmacéuticos Comunitarios. 2014 Jun 01;6(2):33-47. doi:10.5672/FC.21739218.(2014/Nol6).002.06

Autora para correspondencia: Alicia González Rodriguez (aliciagonzalezmeneses@gmail.com). 


\section{KEYWORDS}

osteoarthritis, evaluation of therapy response, clinically significant improvement, drug related problems, negative outcomes associated with medication, community pharmacy, pharmaceutical care
With regard to the quality of life, the most affected activity was it of rising and lowering stairs, presented very much pain when $58(41.1 \%)$ subjects to rise and to lower stairs. We detected 111 negative outcomes associated with medication (NOMs) of those were inefficiency 61 (54.9\%). The pharmaceutical intervention was to offer sanitary education in the selfcare of the joints to $29(20.5 \%)$ patients, recommending physical activity to 32 $(22.7 \%)$ and recommending food-diet-exercise to $23(16.3 \%)$, deriving the specialist to $19(13.4 \%)$ and family doctor to $12(8.5 \%)$.

Conclusion: The community pharmacist's intervention in osteoarthritis patients helps to improve the efficiency of the treatments, improving the results of health of the same ones.

\section{Introducción}

Según la Sociedad Española de Reumatología (SER), la artrosis es una patología articular degenerativa caracterizada por un proceso de deterioro del cartílago, con reacción proliferativa del hueso subcondral e inflamación de la membrana sinovial (1).

Los síntomas que origina son dolor articular, rigidez, inflamación en grado variable y limitación del movimiento. La artrosis puede afectar sólo a una o dos articulaciones, o ser generalizada, con compromiso de varias de ellas. Los sintomas son insidiosos y ceden con el reposo. Después de un período de inactividad prolongado como el sueño, las articulaciones pueden estar rígidas alrededor de treinta minutos. Cuando la enfermedad avanza, el dolor puede ser constante al realizar cualquier actividad física y persistir durante horas después.

Las enfermedades osteoarticulares representan más del 10\% de las consultas (2). Diferentes estudios señalan que entre el 10 y el $40 \%$ de la población general presenta algún trastorno osteoarticular. Las enfermedades osteoarticulares son muy prevalentes en España, especialmente la lumbalgia, la artrosis y la fibromialgia (3-10).

La artrosis es la enfermedad articular más frecuente. Afecta principalmente a las articulaciones que soportan mayor peso y desarrollan un mayor esfuerzo, las localizaciones más frecuentes son cervical, lumbar, caderas, rodillas y las interfalángicas distales.

Según la SER, en España, la osteoartrosis (OA) afecta al 10\% de la población general, representando casi la cuarta parte del total de los pacientes atendidos por los reumatólogos. La frecuencia con que afecta a las personas aumenta con la edad (sobre todo a partir de los 40-50 años), aunque no se debe considerar a esta enfermedad como una consecuencia ineludible del envejecimiento articu- lar (11). Su prevalencia en España en los mayores de 20 años, según el estudio de prevalencia de enfermedades reumáticas en la población española (EPISER) (10), es de un 10,2\% para OA de rodilla, y en los grupos de edad mayores de 60 años, la prevalencia de artrosis sintomática de rodilla asciende al 30\%. Se trata de una de las enfermedades musculoesqueléticas que conllevan una peor calidad de vida por la limitación física que produce. Los determinantes más importantes de la OA sintomática de rodilla en población adulta española son la edad, el sexo femenino y la obesidad, y tiene una gran correlación, además de con el peso, con el estilo de vida y la actividad física (12).

Se estima que entre los pacientes con artrosis de rodilla, uno de cada cuatro está severamente discapacitado (13), lo que produce un coste social y económico de gran magnitud en el mundo occidental por la pérdida de horas laborables, el alto consumo de fármacos y la utilización de diferentes recursos sanitarios. Por todo esto, hay que tener en cuenta que la artrosis es una enfermedad cuyas implicaciones sociales, sanitarias y económicas son muy altas, además es previsible que esto vaya en aumento en las próximas décadas debido al progresivo envejecimiento de la sociedad.

Los objetivos del tratamiento de la OA son: disminuir los síntomas y el dolor, educar al paciente sobre la artrosis, disminuir la discapacidad y prevenir o retardar el progreso de la enfermedad y sus consecuencias. Esto se realiza mediante la utilización de diferentes métodos: tratamiento no farmacológico (educación del paciente, fisioterapia, recomendar ejercicio y cambios en el estilo de vida), tratamiento farmacológico y quirúrgico (lavado articular y artroplastia) (14).

El abordaje farmacológico de la artrosis persigue dos líneas de acción: fármacos que modifican la sin- tomatología: Symptom Modifying Osteo-Arthritis Drugs (SMOADS) y fármacos modificadores de la enfermedad artrósica: Disease Modifying Osteo-Arthritis Drugs (DMOADS). En el grupo de los SMOADS se diferencian dos grupos: fármacos de acción rápida (analgésicos, AINE y glucocorticoides intraarticulares) $\mathrm{y}$ fármacos de acción lenta SYSADOA (Symptomatic Slow Acting Drugs for Osteo-Arthritis). Los principales fármacos SYSADOA son los "precursores de la matriz cartilaginosa" (glucosamina, condroitín sulfato y ácido hialurónico) y los "moduladores de las citoquinas" (diacereína).

Actualmente el tratamiento es sintomático, no existe ningún fármaco con capacidad para frenar la enfermedad artrósica; aunque los síntomas que origina se pueden aliviar, permitiendo que el paciente padezca lo menos posible. Ya que la artrosis no tiene curación definitiva, las estrategias de tratamiento se dirigen a la reducción del dolor, mejora de la movilidad articular así como al mantenimiento de la funcionalidad global de la articulación afectada. A pesar de los tratamientos, la calidad de vida de muchos pacientes con enfermedad osteoarticular crónica disminuye $(3,5,7,9,10,15)$.

La medición objetiva del dolor es un proceso clínico muy complejo, resulta difícil estimar, medir o valorar el dolor que siente una persona. Sin embargo, su cuantificación es muy importante y su evaluación es una actividad clave derivada de una intervención informativa y educativa para ayudar a mejorar la percepción que el paciente tiene sobre su dolor (16).

Para evaluar el dolor, se han desarrollado escalas descriptivas uni y multidimensionales.

Dentro de las unidimensionales la más utilizada es la Escala Visual Analógica (EVA) (17), que destaca por su gran sensibilidad de medición, 
sencillez, versatilidad y manejabilidad estadística, ocupa poco tiempo y es fácil de entender e interpretar por los pacientes. La escala EVA está cuantificada de 0 a $100 \mathrm{~mm}$ (en la que 0 es ausencia de dolor y 100 el nivel máximo de dolor), el paciente anota en la escala el grado de dolor que siente.

Para valorar la función de la articulación se ha empleado el cuestionario de calidad de vida Western Ontario McMaster University Osteoarthritis Index (WOMAC) (18-21). Contiene 24 items agrupados en tres escalas: dolor (5 items), rigidez ( 2 items) y capacidad funcional (17 ítems). Cada ítem se contesta con una escala tipo Likert de 5 niveles que se codifican de la siguiente forma: Ninguno $=0$; Poco $=1$; Bastante $=2 ;$ Mucho = 3; Muchísimo = 4 .

La utilidad del cuestionario WOMAC se basa en la capacidad de evaluar cambios clínicos percibidos por el paciente en su estado de salud como resultado de una intervención.

Por todo ello, el farmacéutico comunitario debe prestar la atención farmacéutica a pacientes con enfermedad artrósica, ya que nos encontramos con pacientes polimedicados, pacientes de edad avanzada, pacientes donde los efectos adversos inducidos por el uso de AINE (22) (hemorragia digestiva, insuficiencia renal, alteraciones plaquetarias, etc.) obligan a elevados costes de prevención, seguimiento y tratamiento, pacientes en los que interese posponer la artroplastia, pacientes en los que otras medidas terapéuticas no hayan sido eficaces, pacientes con afectación exclusiva o casi exclusiva de la rodilla, que no hagan precisar una medicación, y así con la intervención del farmacéutico comunitario se pretende que el medicamento sea efectivo y seguro. Además según las recomendaciones del EULAR 2003 (European League Against Rheumatism) (23) la educación debe formar parte integral del manejo del paciente con artrosis como cualquier otra enfermedad crónica y los profesionales deben informar sobre la evolución de la enfermedad y su manejo terapéutico, ya que diversas técnicas educativas han demostrado reducir el dolor y aumentar las habilidades adquiridas.

No debemos olvidar que el mantenimiento de un tratamiento sintomático, como el de la artrosis, requiere medicamentos eficaces, seguros y cómodos de tomar.
En este entorno de práctica de la atención farmacéutica el farmacéutico comunitario puede implicarse aún más evaluando la efectividad de los tratamientos y solucionando los PRM y RNM que identifique en su práctica profesional. En una patología crónica como la que se está estudiando resulta especialmente interesante analizar si el farmacéutico puede evaluar la respuesta terapéutica a los tratamientos en estos pacientes como indicador de la efectividad de la terapia. Para esta valoración se tiene que tener en cuenta el grado de dolor y el estado funcional de la articulación.

\section{Objetivos}

\section{Objetivo principal}

Evaluar la respuesta terapéutica en pacientes con enfermedad artrósica desde la farmacia comunitaria (FC).

\section{Objetivos específicos}

1. Describir las características farmacoterapéuticas y sociodemográficas de los pacientes con enfermedad artrósica en cinco farmacias comunitarias de Madrid mediante el registro de la dispensación de tratamientos para la osteoartritis.

2. Ofrecer el servicio de seguimiento y educación sanitaria y determinar la proporción de pacientes que alcanzan la respuesta clínica mínima efectiva mediante la medición a través de la escala EVA del dolor antes y después de la intervención y la mejora de la función articular mediante la escala WOMAC antes y después de la intervención.

3. Realizar un análisis descriptivo de los PRM y RNM identificados en pacientes con artrosis, las intervenciones realizadas y el resultado obtenido.

\section{Material y método Diseño}

Estudio cuasi experimental no aleatorizado y multicéntrico realizado en 5 farmacias comunitarias de Madrid durante los primeros 6 meses del 2012.

\section{Sujetos}

La población diana del estudio la constituyeron todos los pacientes que manifestaban tener osteoartrosis y estaban en tratamiento con medicamentos de los grupos terapéuticos analgésicos (N02B), opiáceos (N02AX), AINE (M01A), COXIB (M01AH), otros antiinflamatorios y antirreumáticos no esteroideos (M01AX) y glucocorticoides intraarticulares (H02AB).

La población accesible fueron los pacientes con enfermedad artrósica de la provincia de Madrid que eran usuarios de las 5 farmacias participantes.

La población elegible se determinó por la aplicación a la población accesible de los criterios de selección. Se excluyeron del estudio los pacientes menores de 18 años, por requerir consentimiento de sus progenitores, los pacientes con dificultad de comunicación y aquellos que no aceptaron participar.

La muestra se constituyó sin aleatorización por los pacientes que aceptaron participar, se ofreció participar en el estudio a los pacientes que referían tener artrosis y eran usuarios habituales de las farmacias participantes, durante el mes anterior al inicio del estudio (enero de 2012), informándoles durante la dispensación sobre su tratamiento para la OA.

\section{Procedimiento}

Durante los 6 meses del estudio los farmacéuticos cumplimentaron un cuestionario estructurado (hoja de registro del servicio de dispensación en osteoartrosis) (figura 1), que permitió recoger información sobre el paciente, diagnóstico, medicamentos prescritos, articulaciones afectadas, grado de dolor, estado funcional de la articulación, estado e intervención farmacéutica realizada. Dicho cuestionario permitió cumplir con el primer objetivo específico del estudio: describir las características farmacoterapéuticas y sociodemográficas de los pacientes con enfermedad artrósica.

Para cumplir con el segundo objetivo específico, evaluación de la respuesta terapéutica, se ofreció educación sanitaria, entregando a todos los pacientes un díptico con recomendaciones para el autocuidado en la enfermedad artrósica. Se ofreció el servicio de seguimiento a aquellos pacientes que presentaban inefectividad del tratamiento de la osteoartrosis. Se determinó 


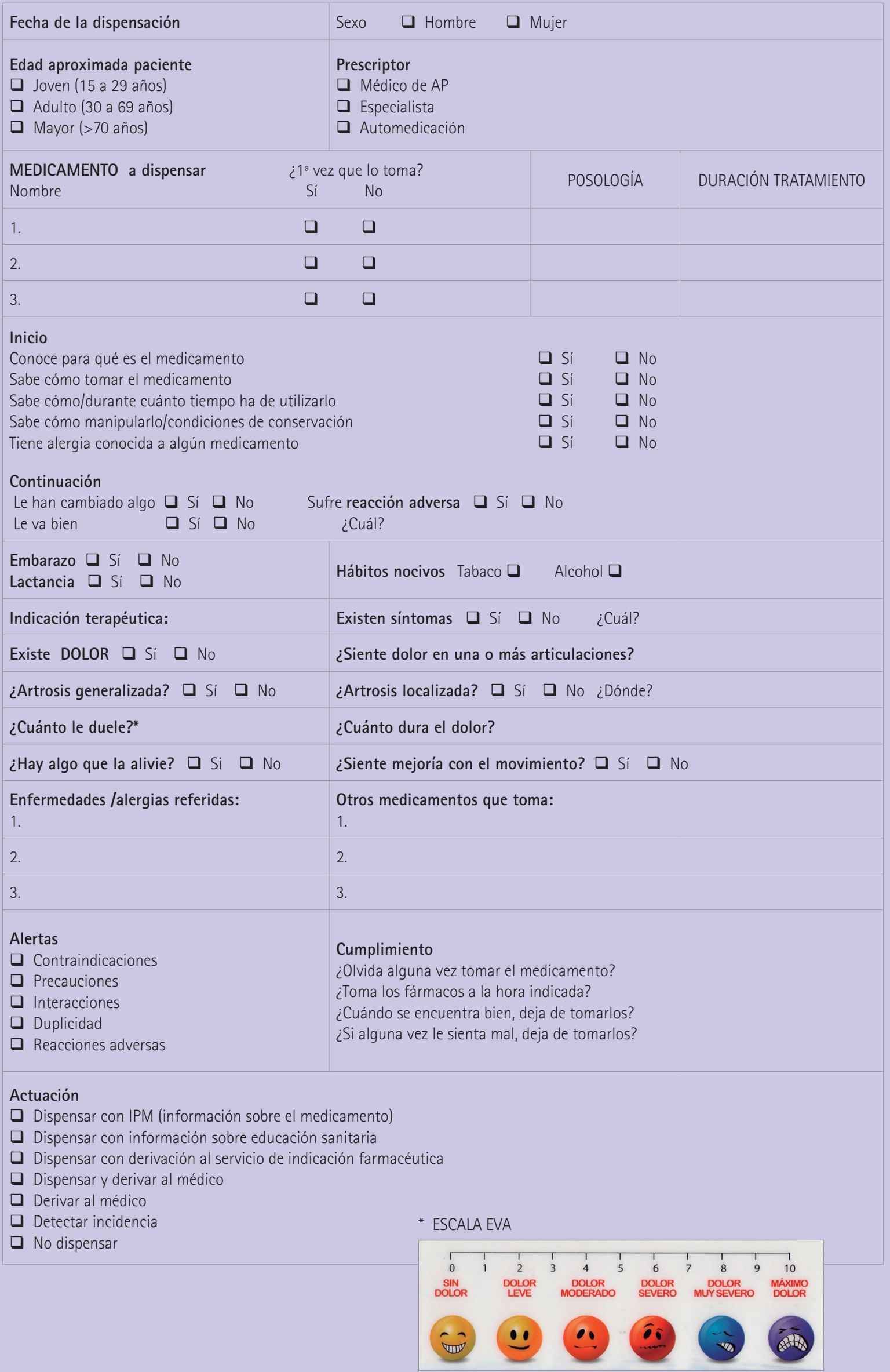

Figura 1 Hoja de registro del servicio de dispensación de osteoartritis y escala visual analógica (EVA) 


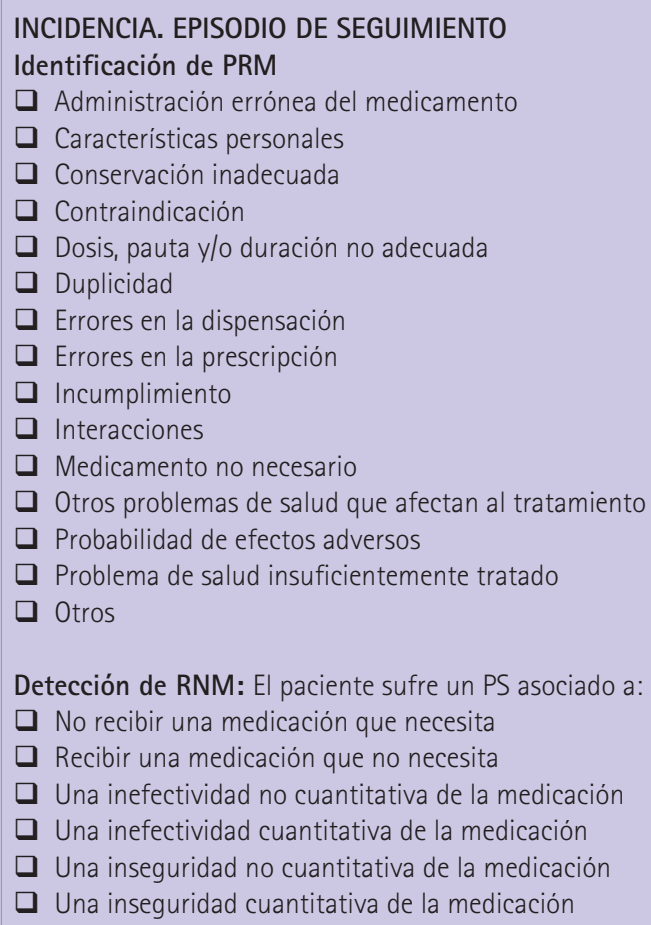

r Riesgo de RNM a RNM Real

Intervención:

- Facilitar información (IPM)

- Ofrecer educación sanitaria

Derivar a seguimiento farmacoterapéutico

- Derivar al médico comunicando el PRM/RNM

- Derivar al médico proponiendo cambios en el tratamiento

Proponer otras modificaciones

Notificar a farmacovigilancia de acuerdo a la legislación vigente

Comunicación:

$\square$ Verbal
$\square$ al médico
$\square$ al paciente $\square$ al farmacéutico
$\square$ Acepta
$\square$ Si otros

Resultado:

Figura 1 (continuación). Hoja de registro del servicio de dispensación de osteoartritis y escala visual analógica (EVA)

la proporción de pacientes que alcanzaron la respuesta clínica mínima efectiva, para lo cual se tuvo en cuenta la propuesta de la Osteo-Arthritis Research Society International (OARSI) para definir la respuesta clínica mínima efectiva, considerándose que una reducción del dolor de $20 \mathrm{~mm}$ en la escala EVA significa que un paciente ha respondido al tratamiento (24). Para evaluar la mejora de la función articular mediante la escala WOMAC antes y después de la intervención, se registraron los cambios percibidos por el paciente en la escala de capacidad funcional, considerando mejoría aceptable cuando el resultado en la escala de capacidad funcional del WOMAC es $\leq 28$. Ambas valoraciones se realizaron en todos los pacientes al inicio, al ser incluidos en el estudio, y al finalizar este, a los 6 meses.

Además, si el farmacéutico detectaba alguna incidencia, la estudiaba y evaluaba, identificando PRM y RNM, clasificándolos según el listado propuesto por Foro de AF en su Documento de Consenso (25), registrando además las intervenciones realizadas y el resultado en salud obtenido, cumpliendo así con el tercer objetivo específico.

\section{Intervención farmacéutica}

Seguimiento Farmacoterapéutico: (metodología Dáder) para aquellos pacientes con inefectividad del tratamiento para su artrosis.

Educación para la Salud (EPS): autocuidado de las articulaciones, realización de ejercicio físico de forma constante y mantenimiento de un peso adecuado, que se ofreció a todos los pacientes incluidos en el estudio.

Para el autocuidado de las articulaciones se informó al paciente sobre las medidas encaminadas a evitar la sobrecarga en las articulaciones, la cual puede ocasionar un aumento del dolor o provocar una progresión más 
rápida de la enfermedad en función de la articulación afectada. Como norma general hay que recomendar que no se realicen los movimientos que producen dolor $\mathrm{y}$, si es posible, se aprenda a usar otras articulaciones en lugar de la enferma. Adoptar una postura adecuada: evitar estar mucho tiempo de pie o mantener la misma postura, dormir en cama dura y sentarse en sillas altas con respaldo, evitar los sillones bajos y hundidos. Evitar sobrecargar la articulación (no caminar cargado, evitar levantar peso). Utilizar calzado adecuado, cómodo, de suela gruesa, plano o con un tacón bajo. Protección articular: el uso de un bastón de apoyo en el brazo contrario a la rodilla o cadera más dolorosa libera parcialmente de peso dicha rodilla durante la marcha. Usar calor (durante 10 minutos para aliviar el dolor) o frío (si hay inflamación).

Se informó sobre la importancia de la actividad física: hacer ejercicio con las articulaciones enfermas es fundamental. El ejercicio debe intentar mantener el movimiento articular y fortalecer los músculos para evitar la flacidez y reducir las rigideces. Debe ser suave y no provocar dolor. Se persigue mejorar la autoestima, entrenar el cuerpo para el desarrollo de actividades, mantener la movilidad articular, frenar la evolución e impedir la aparición de complicaciones, deformidades e incapacidades. Se recomienda caminar por terreno liso y controlando la aparición del dolor, mejor varios paseos cortos que uno largo, si aparece dolor descansar hasta que las molestias se atenúen. Evitar subir y bajar escaleras. Natación y/o ejercicios de movilidad articular en la piscina. Fortalecimiento muscular mediante ejercicios de flexión, extensión con o sin resistencia y estiramientos. Ejercicios de fortalecimiento de cuádriceps especialmente indicados en la artrosis de rodilla, uso de una bicicleta estática con poca resistencia.

Respecto al mantenimiento de un peso adecuado se le transmite que el sobrepeso es perjudicial, especialmente para la evolución de la artrosis de rodilla, ya que estas deben aguantar el peso del cuerpo. Se le recomienda controlar la cantidad de calorías de la ingesta.

Se citaba a los pacientes al cabo de un mes, para comprobar en qué medida eran seguidas las recomen- daciones indicadas, en relación con el autocuidado de las articulaciones, realización de ejercicio físico y mantenimiento de un peso adecuado. Se realizaba una entrevista al paciente en la que se le preguntaba por la adopción de las medidas ofrecidas para el autocuidado articular, así como la realización de los ejercicios propuestos, valorando la presencia o ausencia de dolor durante su realización. A los pacientes con sobrepeso se les propuso realizar dieta y ejercicio y se les realizaba un control del peso, lo que permitía evaluar el resultado de dichas intervenciones.

A los pacientes incluidos en seguimiento, se les citaba mensualmente durante los 6 meses del estudio. Las incidencias detectadas que requerían la intervención del médico eran comunicadas por escrito y se derivaba a los pacientes, registrando la intervención, la respuesta del médico y el resultado en salud obtenido.

\section{Material utilizado}

1. Hoja de registro del servicio de dispensación en osteoartrosis (figura 1).

2. Escala visual analógica (EVA) (17) (figura 1).

3. Cuestionario de calidad de vida Western Ontario McMaster University OsteoarthritisIndex (WOMAC) (18-21) (figura 2).

4. Díptico educativo (figura 3).

\section{Análisis de datos}

Una vez finalizado el período del estudio se llevó a cabo el registro informático de los datos recogidos de todos los pacientes y se procedió al análisis de los mismos. El tratamiento estadístico de los datos se realizó con una hoja de aplicación informática MSExcel ${ }^{\circledR}$.

\section{Resultados}

Durante el periodo de estudio se registraron un total de 141 dispensaciones correspondientes a 205 fármacos. $40(28,3 \%)$ pacientes utilizaban 2 fármacos y $12(8,5 \%) 3$ fármacos. Se observó un predominio de pacientes del sexo femenino, $113(80,1 \%) \mathrm{mu}-$ jeres frente a $28(19,9 \%)$ hombres. En cuanto a la distribución por edades, también se observó un predominio de los grupos etarios de adultos con edades entre 30-69 años, 68 (48,2\%) pacientes, y del grupo mayores de 70 años, 67 (47,5\%) pacientes.

$75(53,2 \%)$ pacientes tenían diagnosticado artrosis localizada y 66 $(46,8 \%)$ pacientes artrosis generalizada, siendo las articulaciones más afectadas: rodillas en 103 (73,0\%) pacientes, vertebras en 71 (50,3\%) y caderas en 53 (37,5\%). Las enfermedades concomitantes mayoritarias referidas por los pacientes fueron: hipertensión arterial, diabetes y osteoporosis.

Los factores de riesgo más prevalentes encontrados fueron: osteoporosis en $28(19,8 \%)$ sujetos, obesidad con $19(13,4 \%)$ personas y enfermedades endocrino-metabólicas en 19 $(13,4 \%)$ pacientes.

Respecto a las manifestaciones clínicas el 100\% de los pacientes presentaba dolor, $89(63,1 \%)$ rigidez articular y 23 (16,3\%) incapacidad funcional.

El tipo de terapia empleada en el tratamiento de las enfermedades artrósicas, así como el tipo de tratamiento farmacológico se describen en la figura 4.

Los fármacos prescritos para la enfermedad artrósica con mayor frecuencia fueron los analgésicos para $51(36,1 \%)$ sujetos y los opiáceos para $33(23,4 \%)$, utilizados con el fin de aliviar el dolor. AINE para $39(27,6 \%)$ sujetos y COXIB para 20 $(14,1 \%)$ para reducir la inflamación y el dolor. Otros antiinflamatorios y antirreumáticos no esteroideos como los precursores de la matriz cartilaginosa $39(27,6 \%)$. Dos $(1,4 \%)$ pacientes estaban tratados con glucocorticoides intraarticulares (figura 4).

Analizando el consumo de fármacos entre los pacientes, se observó que con frecuencia tomaban más de un medicamento, $30(21,2 \%)$ sujetos del estudio tomaban fármacos de los grupos terapéuticos M01A y N02B, 24 (17,0\%) combinaban fármacos de acción rápida y de acción lenta, y 17 (12,0\%) utilizaban también fármacos del grupo terapéutico M02AA.

Respecto a la determinación de la proporción de pacientes que alcanzan la respuesta clínica mínima efectiva mediante la medición a través de la escala EVA del dolor antes y después de la intervención, 34 (24,1\%) pacientes obtuvieron una reducción del dolor de $20 \mathrm{~mm}$ en la escala EVA, 


\section{Cuestionario WOMAC para la artrosis}

\section{Apartado A}

INSTRUCCIONES: Las siguientes preguntas tratan sobre cuánto DOLOR siente usted en las caderas y/o rodillas como consecuencia de su artrosis. Para cada situación indique cuánto DOLOR ha notado en los últimos 2 días. (Por favor, marque sus respuestas con una "X").

\section{PREGUNTA: ¿Cuánto dolor tiene?}

1. Al andar por un terreno llano.

$\begin{array}{ccccc}\square & \square & \square & \square & \square \\ \text { Ninguno } & \text { Poco } & \text { Bastante } & \text { Mucho } & \text { Muchisimo }\end{array}$

2. Al subir y bajar escaleras.

$\begin{array}{ccccc}\square & \square & \square & \square & \square \\ \text { Ninguno } & \text { Poco } & \text { Bastante } & \text { Mucho } & \text { Muchisimo }\end{array}$

3. Por la noche en la cama.

$\begin{array}{ccccc}\square & \square & \square & \square & \square \\ \text { Ninguno } & \text { Poco } & \text { Bastante } & \text { Mucho } & \text { Muchisimo }\end{array}$

4. Al estar senado o tumbado.

$\begin{array}{ccccc}\square & \square & \square & \square & \square \\ \text { Ninguno } & \text { Poco } & \text { Bastante } & \text { Mucho } & \text { Muchisimo }\end{array}$

5. Al estar de pie.

$\begin{array}{ccccc}\square & \square & \square & \square & \square \\ \text { Ninguno } & \text { Poco } & \text { Bastante } & \text { Mucho } & \text { Muchisimo }\end{array}$

\section{Apartado B}

INSTRUCCIONES: Las siguientes preguntas sirven para conocer cuánta RIGIDEZ (no dolor) ha notado en sus caderas y/o rodillas en los últimos 2 dias. RIGIDEZ es una sensación de dificultad inicial para mover con facilidad las articulaciones. (Por favor, marque sus respuestas con una "X").

1. ¿Cuánta rigidez nota después de despertarse por la mañana?

$\begin{array}{ccccc}\square & \square & \square & \square & \square \\ \text { Ninguno } & \text { Poco } & \text { Bastante } & \text { Mucho } & \text { Muchisimo }\end{array}$

2. ¿Cuánta rigidez nota durante el resto del día después de estar sentado, tumbado o descansado?

$\begin{array}{ccccc}u & \square & \square & \square & \square \\ \text { Ninguno } & \text { Poco } & \text { Bastante } & \text { Mucho } & \text { Muchisimo }\end{array}$

\section{Apartado C}

INSTRUCCIONES: Las siguientes preguntas sirven para conocer su CAPACIDAD FUNCIONAL. Es decir, su capacidad para moverse, desplazarse o cuidar de si mismo. Indique cuanta dificultad ha notado en los últimos dos dias al realizar cada una de las siguientes actividades, como consecuencia de su artrosis de cadera y/o rodillas. (Por favor, marque sus respuestas con una "X").

PREGUNTA: ¿Qué grado de dificultad tiene al...?

1. Bajar las escaleras.

$\begin{array}{ccccc}\square & \square & \square & \square & \square \\ \text { Ninguno } & \text { Poco } & \text { Bastante } & \text { Mucho } & \text { Muchisimo }\end{array}$

2. Subir las escaleras.

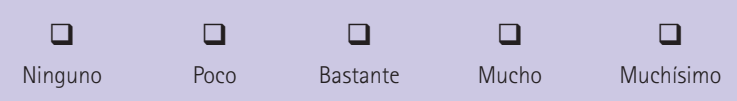

3. Levantarse después de estar sentado.

$\begin{array}{ccccc}\square & \square & \square & \square & \square \\ \text { Ninguno } & \text { Poco } & \text { Bastante } & \text { Mucho } & \text { Muchisimo }\end{array}$

Figura 2 Cuestionario de calidad de vida WOMAC 
4. Estar de pie.

$\begin{array}{ccccc}\square & \square & \square & \square & \square \\ \text { Ninguno } & \text { Poco } & \text { Bastante } & \text { Mucho } & \text { Muchisimo }\end{array}$

5. Agacharse para coger algo.

$\begin{array}{ccccc}\square & \square & \square & \square & \square \\ \text { Ninguno } & \text { Poco } & \text { Bastante } & \text { Mucho } & \text { Muchisimo }\end{array}$

6. Andar por un terreno llano.

$\begin{array}{ccccc}\square & \square & \square & \square & \square \\ \text { Ninguno } & \text { Poco } & \text { Bastante } & \text { Mucho } & \text { Muchisimo }\end{array}$

7. Entrar y salir de un coche.

$\begin{array}{ccccc}\square & \square & \square & \square & \square \\ \text { Ninguno } & \text { Poco } & \text { Bastante } & \text { Mucho } & \text { Muchisimo }\end{array}$

8. Ir de compras.

$\begin{array}{ccccc}\square & \square & \square & \square & \square \\ \text { Ninguno } & \text { Poco } & \text { Bastante } & \text { Mucho } & \text { Muchisimo }\end{array}$

9. Ponerse las medias o los calcetines.

$\begin{array}{ccccc}\square & \square & \square & \square & \square \\ \text { Ninguno } & \text { Poco } & \text { Bastante } & \text { Mucho } & \text { Muchisimo }\end{array}$

10. Levantarse de la cama.

$\begin{array}{ccccc}\square & \square & \square & \square & \square \\ \text { Ninguno } & \text { Poco } & \text { Bastante } & \text { Mucho } & \text { Muchisimo }\end{array}$

11. Quitarse las medias o los calcetines.

$\begin{array}{ccccc}\square & \square & \square & \square & \square \\ \text { Ninguno } & \text { Poco } & \text { Bastante } & \text { Mucho } & \text { Muchisimo }\end{array}$

12. Estar tumbado en la cama.

$\begin{array}{ccccc}\square & \square & \square & \square & \square \\ \text { Ninguno } & \text { Poco } & \text { Bastante } & \text { Mucho } & \text { Muchisimo }\end{array}$

13. Entrar y salir de la ducha/bañera.

$\begin{array}{ccccc}\square & \square & \square & \square & \square \\ \text { Ninguno } & \text { Poco } & \text { Bastante } & \text { Mucho } & \text { Muchisimo }\end{array}$

14. Estar sentado.

$\begin{array}{ccccc}\square & \square & \square & \square & \square \\ \text { Ninguno } & \text { Poco } & \text { Bastante } & \text { Mucho } & \text { Muchisimo }\end{array}$

15. Sentarse y levantarse del retrete.

$\begin{array}{ccccc}\square & \square & \square & \square & \square \\ \text { Ninguno } & \text { Poco } & \text { Bastante } & \text { Mucho } & \text { Muchisimo }\end{array}$

16. Hacer tareas domésticas pesadas.

$\begin{array}{ccccc}\square & \square & \square & \square & \square \\ \text { Ninguno } & \text { Poco } & \text { Bastante } & \text { Mucho } & \text { Muchisimo }\end{array}$

17. Hacer tareas domésticas ligeras.

$\begin{array}{ccccc}\square & \square & \square & \square & \square \\ \text { Ninguno } & \text { Poco } & \text { Bastante } & \text { Mucho } & \text { Muchisimo }\end{array}$



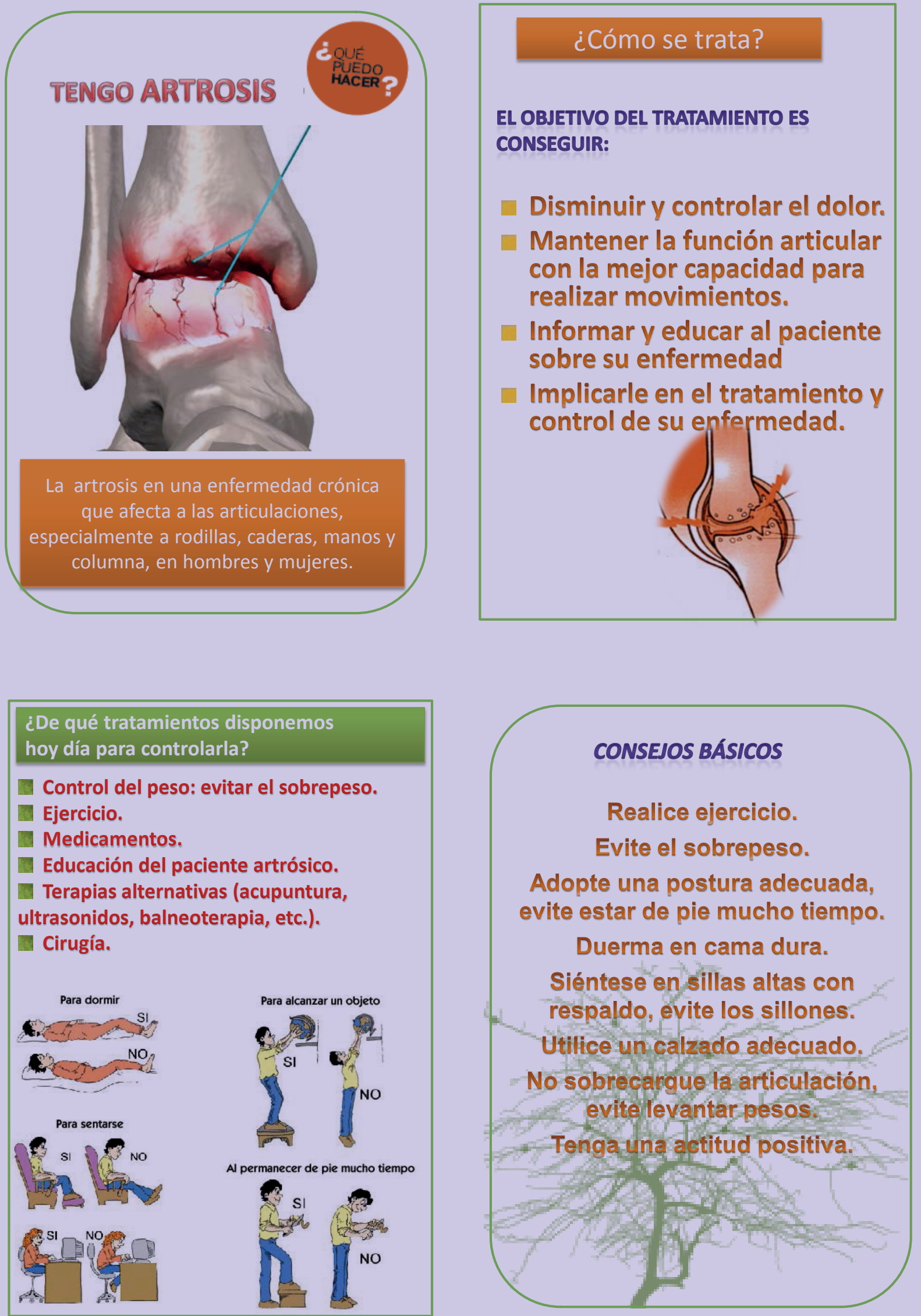


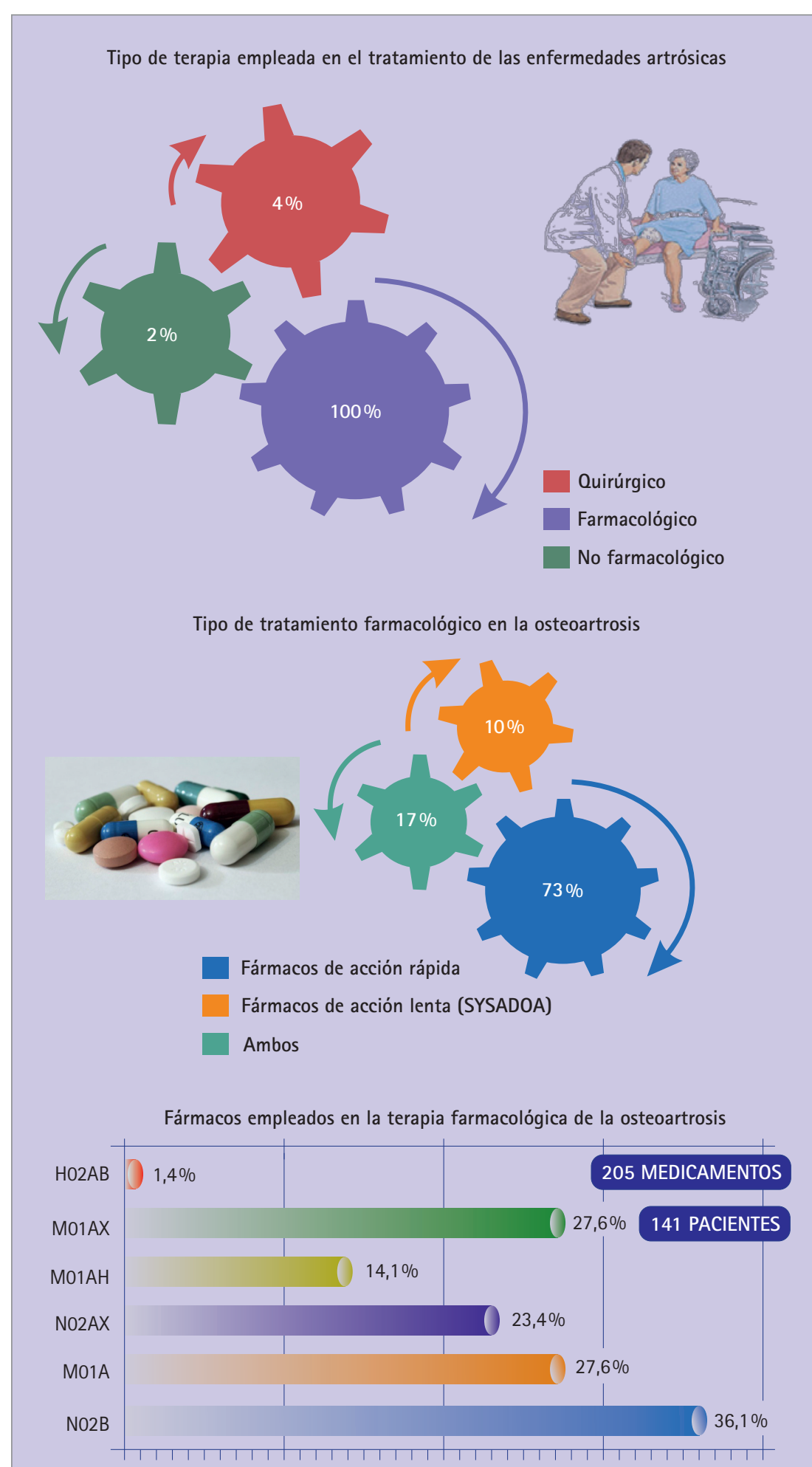

H02AB: glucocorticoides intraarticulares. M01AX: otros antiinflamatorios y antirreumáticos no esteroideos. M01AH: coxibs. M01A: AINE. N02AX: opiáceos. N02B: analgésicos.

Figura 4 Terapias y tratamientos empleados en la artrosis

alcanzando así la respuesta clínica mínima efectiva; mientras que $3(2,1 \%)$ pacientes sufrieron un agravamiento en el grado de dolor y 104 (73,8\%) pacientes no presentaron cambios en el grado de dolor. En la figura 5 se representa la distribución de los pa- conseguido una reducción del dolor tras la intervención.

Al final 7 (5,0\%) pacientes presentan un grado de dolor leve (EVA: 2) y $32(23,0 \%)$ pacientes un grado de dolor moderado (EVA: 4), este incremento de paciente con dolor leve y moderado tras la intervención se corresponde con 7 (5,0\%) pacientes que al inicio presentaban un grado de dolor severo (EVA: 6) y han conseguido una reducción del dolor.

En las figuras 6 y 7 se representan los resultados en las diferentes subescalas del cuestionario WOMAC antes y después de las intervenciones. Se pueden observar los cambios percibidos por los pacientes en su estado de salud como resultado de las intervenciones.

Respecto a la calidad de vida, la actividad más afectada fue la de subir y bajar escaleras, presentado muchísimo dolor al hacerlo (W2) 53 (37,7\%) pacientes antes de la intervención y $50(35,5 \%)$ tras la intervención; presentaron muchísima dificultad al subir escaleras (W9) 59 (41,8\%) pacientes antes de la intervención y 50 (35,5\%) después de la intervención; muchísima dificultad al bajar escaleras (W8) 61 (43,3\%) antes de la intervención y $50(35,5 \%)$ tras la intervención. También presentaron dificultad para la realización de tareas domésticas pesadas (W16) 51 (36,2\%) pacientes antes de la intervención y 45 (31,9\%) después de la intervención (figura 7), suponiendo estos cambios un aumento en la calidad de vida de los pacientes.

La puntuación obtenida en la escala de capacidad funcional del WOMAC antes de las intervenciones fue $106(75,2 \%)$ pacientes con puntuación >28 y $35(24,8)$ pacientes con puntuación $\leq 28$; mientras que tras la intervención fue $36(25,5 \%)$ pacientes con puntuación >28 y 105 (74,5\%) pacientes con puntuación $\leq 28$, por lo que los pacientes con mejoría en la capacidad funcional tras la intervención son 70 (49,6\%).

En la figura 8 se representa la distribución de los PRM detectados durante el estudio (117 PRM). Incluidos en otros PRM 7 (9,6\%) son debidos a automedicación con AINE ocasionando aumento de los valores de tensión arterial. Se detectaron un total de 111 RNM, de los cuales 61 (54,9\%) fueron de inefectividad y $49(44,1 \%)$ de inseguridad (figura 9). cientes de acuerdo con el grado de dolor que padecian antes y después de las intervenciones. Se puede observar que al inicio 52 (37,0\%) pacientes presentaban dolor muy severo (EVA: 8), y al final se redujo a 28 (20,0\%), con lo que $24(17,0 \%)$ pacientes han 
Distribución de pacientes según el grado de dolor antes de la intervención

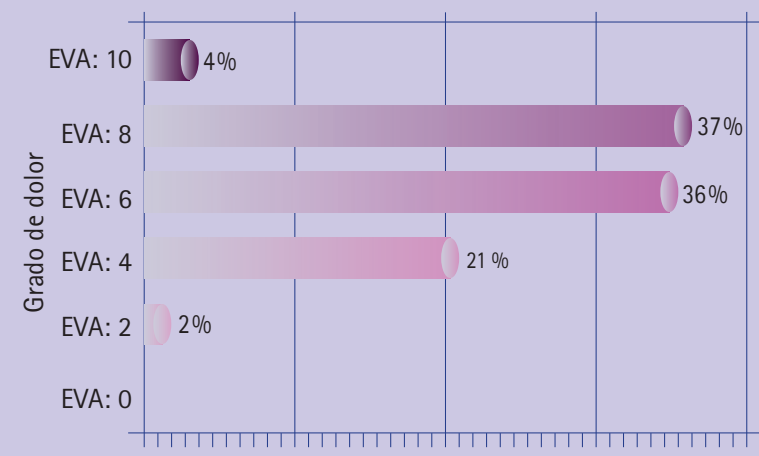

$\%$ pacientes
Distribución de pacientes según el grado de dolor después de la intervención

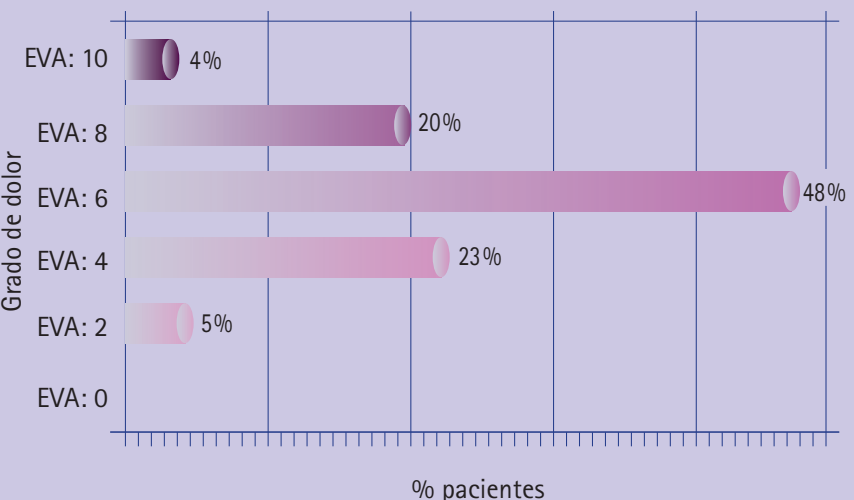

Figura 5 Distribución de los pacientes según el grado de dolor con la escala visual analógica (EVA)

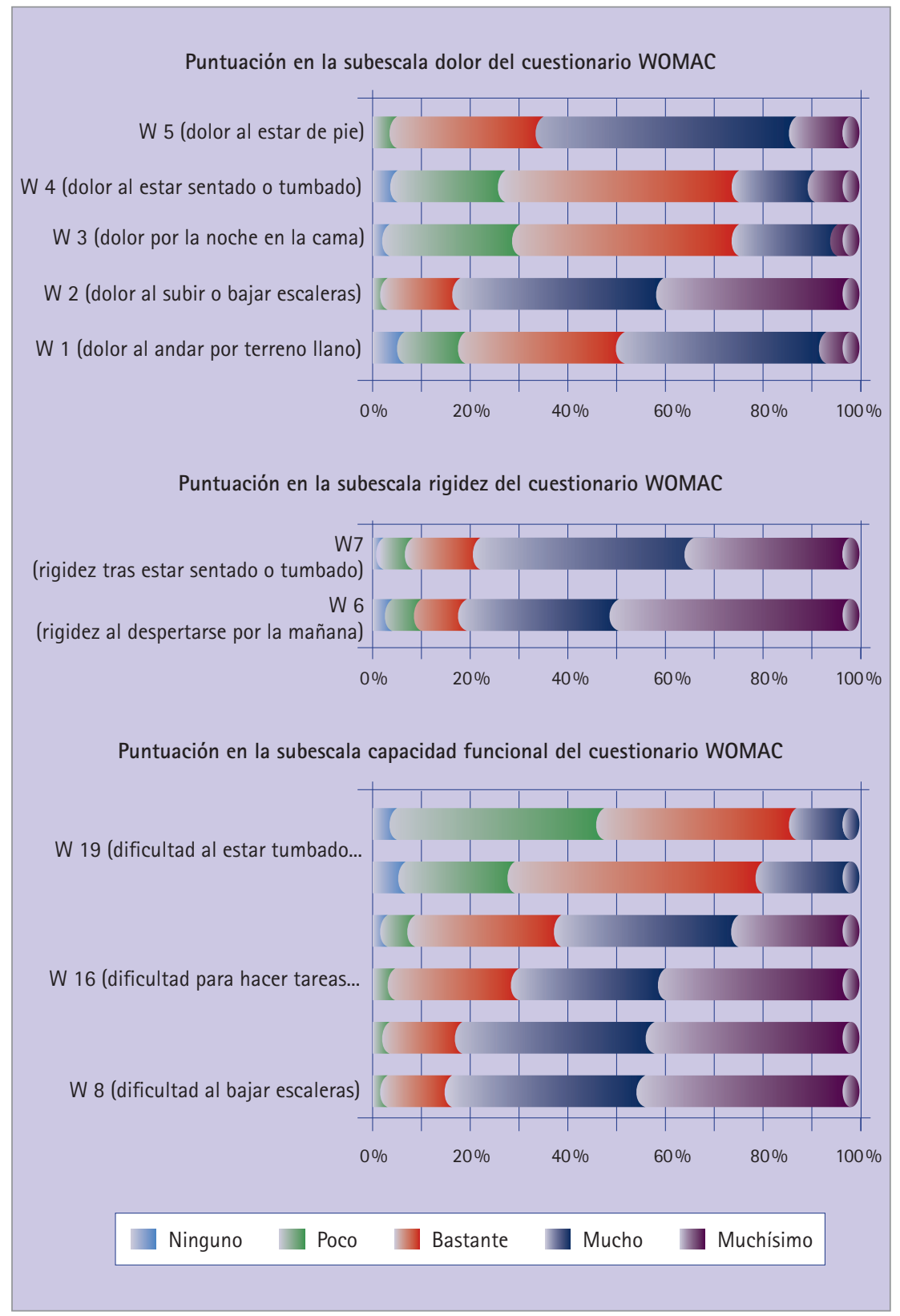

Figura 6 Evaluación del cuestionario WOMAC antes de la intervención
Se intervino ofreciendo educación sanitaria en el autocuidado de las articulaciones a toda la muestra del estudio y de manera específica se recomendó ejercicio físico a 32 (22,7\%), dieta-ejercicio a 23 (16,3\%) con sobrepeso, se ofreció seguimiento a 61 (54,9\%) pacientes, se derivó al especialista a $19(13,4 \%)$ y al MAP a $12(8,5 \%)$.

Las intervenciones propuestas por el farmacéutico fueron aceptadas por $133(94,3 \%)$ pacientes. A 5 (3,5\%) que no aceptaron nuestra intervención se les había recomendado ejercicio físico y 3 (2,2\%) pacientes con sobrepeso tampoco aceptaron la intervención del farmacéutico, que les proponía dieta-ejercicio. Todas las intervenciones propuestas al MAP, relativas a inseguridad del tratamiento, fueron aceptadas, así como las intervenciones propuestas al especialista relacionadas con inefectividad de la medicación.

Los resultados obtenidos en el estado de salud con la valoración de la respuesta terapéutica fueron: mejoría alcanzando la respuesta clínica minima efectiva $34(24,1 \%)$ pacientes, mejoría parcial con mejoría en la capacidad funcional (no se alcanza una reducción del dolor de $20 \mathrm{~mm}$ en la EVA pero sí hay mejora de la capacidad funcional en el WOMAC) en 39 (27,6\%), sin cambios 59 (41,8\%) de los sujetos, se desconoce 8 (5,7\%) y empeoraron 3 (2,1\%), determinados según la reducción del dolor en la escala EVA y la mejora en la función articular con el cuestionario WOMAC. 


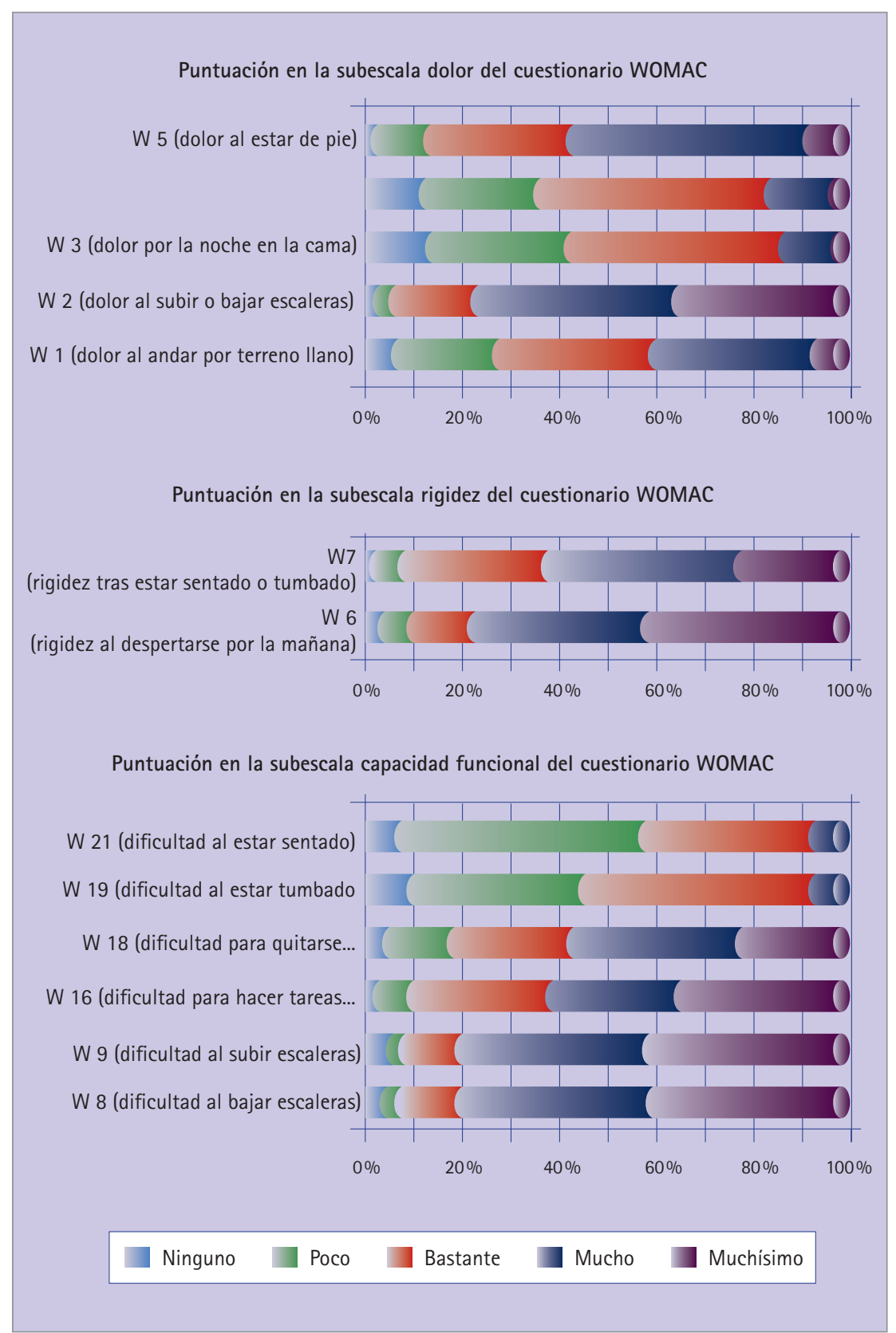

Figura 7 Evaluación del cuestionario WOMAC después de la intervención

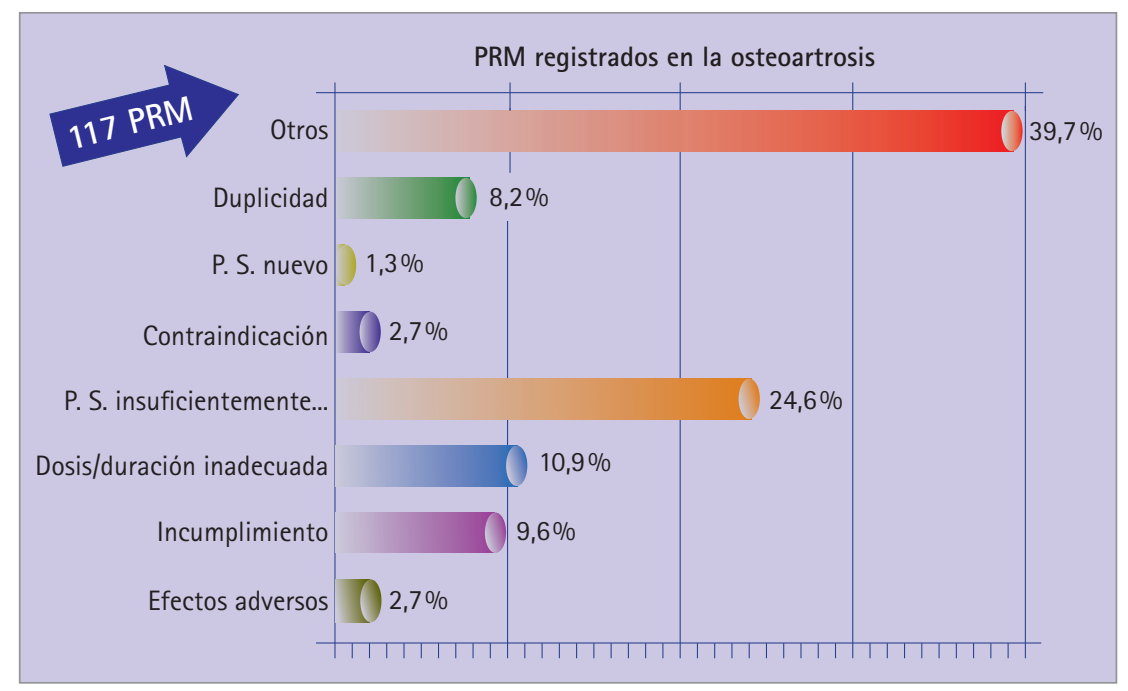

Figura 8 PRM detectados

\section{Discusión}

Tras el estudio realizado sobre la evaluación de la respuesta terapéutica en pacientes con enfermedad artrósica por farmacéuticos comunitarios se observan diferencias según sea el sexo del paciente, siendo en esta patología las mujeres más propensas a padecerla que los hombres. Son los propios pacientes, en su mayoría, los que acuden a las farmacias comunitarias solicitando el tratamiento prescrito. Nuestros datos coinciden con las cifras de mayor prevalencia de la enfermedad en las mujeres, como muestra el estudio epidemiológico EPISER del año 2000, promovido por la Sociedad Española de Reumatología (8).

Respecto al tipo de terapia empleada en el tratamiento de las enfermedades artrósicas, en el 4\% de los pacientes de nuestro estudio, que presentaba una artrosis avanzada e incapacitante, fue necesaria la cirugía para atenuar el dolor y recuperar la mayor función posible de la articulación; el 100\% de los pacientes utiliza medicamentos para modificar la sintomatología; y un porcentaje muy pequeño de los pacientes se ayuda de terapias no farmacológicas, pese a que las medidas farmacológicas resultan más efectivas cuando se combinan con medidas no farmacológicas que deberían mantenerse siempre para el control de la enfermedad. No debemos olvidar que el plan de tratamiento para la artrosis incluye, además de los medicamentos, ejercicio, control de peso, descanso y cuidado de las articulaciones, técnicas para controlar el dolor sin fármacos y terapias complementarias.

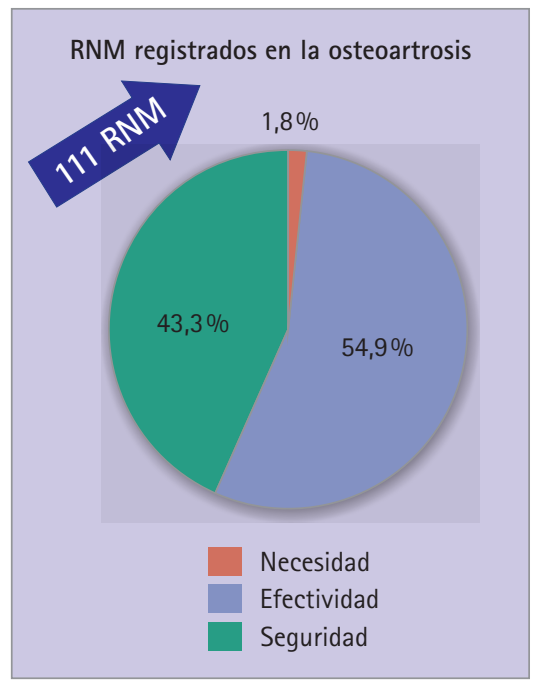

Figura 9 RNM detectados 
Los medicamentos más prescritos son los analgésicos, siguiendo las recomendaciones de los expertos que indican como primer tratamiento analgésico el paracetamol en dosis de $1 \mathrm{~g}$, siempre que la elección se fundamente en decisiones individualizadas ante cada paciente (26), seguido de los AINE, opiáceos y los precursores de la matriz cartilaginosa.

En nuestro estudio el 75,9\% de los pacientes no obtuvo una reducción del dolor de $20 \mathrm{~mm}$ en la escala EVA, por lo que no alcanzan la respuesta clínica mínima efectiva necesaria para considerar que han respondido al tratamiento, lo que nos hace pensar en una falta de efectividad.

El cuestionario de calidad de vida WOMAC es una herramienta de gran utilidad para evaluar los cambios clínicos percibidos por el paciente en su estado de salud, que el farmacéutico comunitario debe conocer y utilizar, y nos permite valorar la función articular. En el estudio ARTROCAD se informa que la mitad de los encuestados necesitaba algún tipo de ayuda para realizar las actividades cotidianas más básicas y un tercio de esta población pasaba más de 8 horas al día sentada (15). En nuestra muestra al inicio un elevado porcentaje de pacientes presentaba limitaciones y dolor a la hora de realizar determinadas actividades (las más afectadas subir y bajar escaleras y la realización de tareas domésticas pesadas) afectando a su calidad de vida, tras las intervenciones se consiguió un aumento de la calidad de vida tras reducirse el porcentaje de pacientes con limitaciones y dolor.

Durante el estudio se han detectado PRM relacionados con la dosis/ duración inadecuada, problema de salud insuficientemente tratado, incumplimiento, duplicidad, y otros (tolerancia que producen los analgésicos opiáceos N02AX prescritos en la enfermedad artrósica) relativos a una falta de eficacia del tratamiento. También fueron muy significativos los PRM registrados como "otros", relacionados con la automedicación, específicamente de los AINE, que ocasionaban aumento de los valores de tensión arterial en los sujetos que los toman para el tratamiento de la enfermedad artrósica, relativos a una falta de seguridad que provocan dichos fármacos. Los antiinflamatorios no esteroideos se encuentran entre los fármacos más consumidos del mundo. En 2012 la Agencia Española de Medicamentos y Productos Sanitarios (AEMPS) emitió la nota informativa 15/2012 según la cual el balance beneficio riesgo de los AINE se mantiene positivo, pero deben utilizarse a la menor dosis y durante el menor tiempo posible(27).

En cuanto a los RNM registrados en el estudio se debieron en su mayoría a una inefectividad de los tratamientos, y también por inseguridad de los mismos. No debemos olvidar que el mantenimiento de un tratamiento sintomático, como el de la artrosis, requiere medicamentos eficaces, seguros y cómodos de tomar. Este hecho pone de manifiesto el importante papel que desempeña el farmacéutico comunitario en la optimización del tratamiento terapéutico de los pacientes y en la detección e identificación de los PRM, para prevenir y resolver RNM de falta de efectividad y falta de seguridad de los mismos, que real o potencialmente pudieran aparecer.

Nuestra actuación sobre los factores de riesgo modificables es de vital importancia para prevenir y enlentecer las alteraciones del cartílago articular. Es recomendable que los farmacéuticos enseñemos a los pacientes cómo afrontar la enfermedad artrósica, haciendo hincapié en el autocuidado de la salud, mantenimiento de un peso adecuado y realización de ejercicio físico de forma constante. En nuestro estudio se ofreció a los pacientes obesos un programa que incluía consejo dietético y ejercicio aeróbico, ya que la reducción de peso junto con el ejercicio ha demostrado reducir el dolor y mejorar la funcionalidad.

La mayoría de las intervenciones propuestas por los farmacéuticos comunitarios fueron aceptadas, obteniendo como resultado una mejoría alcanzando la respuesta clínica mínima efectiva $34(24,1 \%)$ pacientes, y mejoría parcial (no se alcanza una reducción del dolor de $20 \mathrm{~mm}$ en la EVA pero sí hay mejora de la capacidad funcional en el WOMAC) 39 $(27,6 \%)$ pacientes.

Disponer de un cuestionario para la artrosis que valore aspectos de funcionalidad de las articulaciones y calidad de vida es de gran interés para su aplicación en la atención farmacéutica. En nuestro estudio, al igual que en otros trabajos $(21,28,29)$, el cuestionario de calidad de vida WOMAC ha mostrado ser un instrumento válido, seguro y fiable para evaluar los cambios clínicos percibidos por el paciente en su estado de salud, además de aplicable en la farmacia comunitaria a cualquier paciente con OA. Este índice se usa en estudios para la valoración de la efectividad de diferentes procedimientos terapéuticos, pero también sirve para monitorizar la enfermedad. El inconveniente que tiene para su implementación es el tiempo que requiere su utilización e interpretación.

No hemos encontrado ningún trabajo con esta metodología realizado en FC. En el estudio de Núñez y col (28) se evalúa el efecto de la educación terapéutica y la readaptación funcional en la calidad de vida en pacientes con diagnóstico de artrosis en una lista de espera para prótesis total de rodilla. Se trata de un ensayo controlado aleatorizado con una duración de 9 meses, 80 pacientes completaron el estudio, se encontró una mejora en la función articular según el WOMAC para el grupo intervención con respecto al grupo control. Por el contrario, en nuestro estudio en FC no se realizo aleatorización, finalizaron el estudio 141 pacientes y la duración fue de 6 meses; igualmente encontramos una mejora en la función articular en aquellos pacientes en los que el farmacéutico realizó una intervención de educación para la salud (autocuidado de las articulaciones, mantenimiento de un peso adecuado y realización de ejercicio físico de forma constante).

En el estudio anteriormente citado (28) la dimensión de la función medida por WOMAC de los pacientes que recibieron tanto el tratamiento farmacológico y educación terapéutica y la readaptación funcional mejoró con respecto al grupo de control que recibió sólo el tratamiento farmacológico. Esto sugiere que un programa de educación terapéutica y la readaptación funcional durante el período en lista de espera para el reemplazo total de rodilla puede reducir el impacto negativo de esta situación. En nuestro estudio también se observó una mejora en la dimensión de la función medida por WOMAC en los pacientes que recibieron EPS, mejorando así los resultados en salud de estos pacientes. Se pretende que los beneficios de 
una intervención farmacéutica en la salud del paciente sean para todos los pacientes con enfermedad osteoartrósica, no solamente para los pacientes que estén en lista de espera para el reemplazo total de rodilla.

Consideramos limitación del presente estudio el reducido tamaño muestral teniendo en cuenta la alta prevalencia de las enfermedades OA. Creemos que una muestra más amplia permitiría mostrar una mayor contribución de la intervención farmacéutica en los resultados en salud de los pacientes con artrosis. También supone una limitación el periodo de tiempo de estudio, pues hace falta mayor tiempo para poder observar cambios en la salud del paciente tras la intervención. Otra limitación del estudio es la no existencia de grupo control, por lo que las mejoras obtenidas no se pueden atribuir de manera categórica a la intervención del farmacéutico.

Es importante resaltar que las herramientas empleadas (EVA y WOMAC) en este trabajo son un método útil y sencillo para valorar la respuesta terapéutica en pacientes con artrosis en las farmacias comunitarias y pueden ser usadas en la práctica diaria, y no únicamente en ensayos clínicos, ya que permiten una adecuada evaluación del paciente con artrosis y una valoración del impacto de cualquier intervención terapéutica que se aplique.

Realizando una evaluación de la respuesta terapéutica en pacientes con enfermedad artrósica se colabora con el equipo multidisciplinar, contribuyendo al cumplimiento de los objetivos que se persigue con el tratamiento (controlar el dolor, mejorar el funcionamiento de la articulación, mantener un peso saludable y retrasar la progresión de la enfermedad).

Además, el farmacéutico comunitario desempeña un papel fundamental en la detección e identificación de PRM, previniendo y/o evitando RNM que real o potencialmente puedan aparecer.

\section{Agradecimientos}

Queremos agradecer a nuestros mentores en atención farmacéutica: la Doctora en Farmacia Pura Lledó Polo, el Doctor en Farmacia Ramón Sánchez de Rojas Fernández-Cabrera, y $D^{a}$ Raquel Varas. También a los titulares de las farmacias cominitarias donde se realizó nuestro trabajo: $\mathrm{D}^{\mathrm{a}}$ Antonia Hijas Mirón, Da Milagros Gutiérrez Payo, D José Luis Rey de Viñas Rodríguez, Da Gloria Vázquez Salgueiro y $\mathrm{D}^{\mathrm{a}}$ Florinda González Quintas, así como a todos los pacientes que participaron en el estudio.

\section{Referencias bibliográficas}

1. Alonso A. Batlle-Gualda E, Benito P, Blanco F, Martin E. Manual SER de la Artrosis. Artrosis: definición y clasificación. Madrid: IMctC; 2002. p.9.

2. Cueto Espinar A, Hernández Mejía R, Fernández López JA, Ballina García FJ. Enfermedades del sistema osteomuscular y tejido conjuntivo. En: Piédrola Gil G, Del Rey Calero J, Cortina Greus P, Gálvez Vargas R, Sierra López A, Sáenz González MC, et al. Medicina preventiva y salud pública. Barcelona: Masson; 2008. p. 885-896.

3. Loza E, Abasolo L, Jover JA, Carmona L. Burden of disease across chronic diseases: a health survey that measured prevalence, function, and quality of life. J Rheumatol 2008;5:159165.

4. Loza E, Jover JA, Rodriguez-Rodriguez L, Carmona L. EPISER Study Group. Observed and expected frequency of co-morbid chronic diseases in rheumatic patients. Ann Rheum Dis. 2008;67:418-421. doi:10.1136/ ard.2007.078659

5. Loza E, Jover JA, Rodríguez Y, Carmona L. EPISER Study Group. Multimorbidity: prevalence, effect on quality of life and daily functioning, and variation of this effect when one condition is a rheumatic disease. Semin Arthritis Rheum. 2008;38:312-319. doi:10.1016/j.semarthrit.2008.01.004

6. Fernández-López JC, Laffon A, Blanco FJ, Carmona L. Prevalence, risk factors, and impact of knee pain suggesting osteoarthritis in Spain. Clin Exp Rheumatol. 2008; 26: 324-332.

7. Mas AJ, Carmona L, Valverde M, Ribas B. Prevalence and impact of fibromyalgia on function and quality of life in individuals from the general population: results from a nationwide study in Spain. Clin Exp Rheumatol. 2008;26:519-526.

8. Carmona L, Villaverde V, Hernández-García C, Ballina J, Gabriel R, Laffon A. EPISER StudyGroup. The prevalence of rheumatoid arthritis in the general population of Spain. Rheumatology (Oxford). 2002;41:88-95. doi:10.1093/rheumatology/41.1.88

9. Carmona L, Ballina FJ, Gabriel R, Laffon A. EPISER Study Group. The burden of musculoeskeletal diseases in the general population of Spain: results from a nation-wide study. Ann Rheum Dis. 2001;60:1040-1045. doi:10.1136/ard.60.11.1040

10. Carmona L, Gabriel R, Ballina FJ, Laffon A. Grupo de Estudio EPISER. Proyecto EPISER 2000: prevalencia de enfermedades reumáticas en la población española. Metodología, resultados del reclutamiento y características de la población. Rev Esp Reumatol. 2001;28:18-25.

11. Benito-Ruiz P. Artrosis. Etiopatogenia, epidemiología y clasificación. En: Blanco García FJ, Carreira Delgado P, Martín Mola E, Mulero Mendoza J, Navarro Sarabia F, Olivé Marqués A, et al. (editores). Manual SER de las enfermedades reumáticas. Madrid: Médica Panamericana $4^{\mathrm{a}} \mathrm{Ed}$; 2004. p. 315-9.

12. De Filippis L, Gulli S, Carliri A.Gruppo OASIS (Osteoarthritis South Italy Study). Epidemiology and risk factors in osteoarthritis: literature review data from "OASIS" study. Reumatismo. 2004;56(3):169-184.

13. Peat G, McCarney R, Croft P. Knee pain and osteoarthritis in older adults: a review of community burden and current use of health care. Ann Rheum Dis. 2001;60:91-97. doi:10.1136/ard.60.2.91

14. Toquero F, Zarco J, Möller I, Giménez S, Pulido F.J, Trigueros J.A. Atención Primaria de Calidad. Guía de Buena Práctica Clínica en Artrosis. Madrid: Ministerio de Sanidad y Consumo. IMEC； 2004. ISBN: 84-689-0275-6 65-101.

15. Batlle-Guada E, Carmona L, Garrila D, García Criado EI, Ruiz R, Carbonell J. Grupo ArtRoCad.Implementación y características de la población del estudio ArtRoCad, una aproximación al consumo de recursos y repercusión socioeconómica de la artrosis de rodilla y cadera en atención primaria. Reumatol Clin. 2006;2:224-234.

16. Ballina J, Carmona L, Laffon A. Grupo de Estudio EPISER. Impacto del consumo de AINE en la población general española: resultados del estudio EPISER. Rev Esp Reumatol. 2002;29:337-342.

17. Jordan KM, Arden NK, Doherty M, Bannwarth B, Bijlsma JW, Dieppe $\mathrm{P}$, et al. EULAR Recommendations 2003: anevidencebasedapproach to themanagement of kneeosteoarthritis: Report of a Task Force of the Standing Committee for International Clinical Studies Including Therapeutic Trials (ESCISIT). Ann Rheum Dis. 2003;62;1145-1155. doi:10.1136/ ard.2003.011742

18. Doukkali A, Murillo D, Motilva V, Lacalle JR. Intervención farmacéutica y percepción del paciente sobre su dolor. Farmacéuticos Comunitarios. 2011 Sep 30;2(3):93-99. 
19. Huskisson EC, Jones J, Scott PJ. Application of visual-analogue scales to the measurement of functional capacity. Rheumatol Rehabil. 1976;15:185-187. doi:10.1093/rheumatology/15.3.185

20. Dawson J, Carr A. Outcomes evaluation in orthopaedics. J Bone Joint Surg (Br). 2001;83-B(3):313-315. doi:10.1302/0301-620X.83B3.12148

21. Bellamy N, Buchanan WW, Goldsmith CH, Campbell J, Stitt LW, et al. Validation study of WOMAC: A health status instrument for measuring clinically important patient relevant outcomes to antirheumatic drug therapy in patients with osteoarthritis of the hip or knee. J Rheumatol. 1988;15:1833-1840.

22. Bellamy N. Pain assessment in osteoarthritis: Experience with the WOMAC osteoarthritis index. Semin Arthritis Rheum. 1989;18(Supl.2):14-17.

23. Escobar A, Quintana JM, Bilbao A, Azkárate J, Guenaga JI. Validation of the Spanish version of the WOMAC questionnaire for patients with hip or knee osteoarthritis. Western Ontario and McMaster Universities Osteoarthritis Index. Clin Rheumatol. 2002;21:466-471. doi:10.1007/ s100670200117

24. Dougados M, Leclaire P, Van der Heiijde D, Bloch DA, Bellamy N, Altman $\mathrm{RD}$. Response criteria for clinical trials on osteoarthritis of the knee and hip: a report of the Osteoarthritis Research Society International Standing Committee for Clinical Trials Response Criteria Initiative. Osteoarthritis Cartilage. 2000;8:395-403. doi:10.1053/ joca.2000.0361

25. Foro de Atención Farmacéutica en Farmacia Comunitaria, panel de expertos. Guía práctica para los servicios de atención farmacéutica en la farmacia comunitaria. Madrid: Consejo General de Colegios Oficiales de Farmacéuticos; 2010. ISBN 13: 97884-693-1717-4.

26. Panel de expertos de la Sociedad Española de Reumatología (SER). Primer documento de consenso de la Sociedad Española de Reumatología sobre el tratamiento farmacológico de la artrosis de rodilla. Reumatol Clin 2005; 1: 38-48.

27. Caelles N, Silva MM. Efectividad, seguridad y uso de ibuprofeno no sujeto a prescripción médica. Farmacéuticos Comunitarios. 2013 Dec 19;5(4):152162.

28. Núñez M, Núñez E, Segur JM, Macule F, Quinto L, Hernández MV, et al.The effect of an educational program to improve health-related quality of life in patients with osteoarthritis on waiting list for total knee replacement: a randomized study. Osteoarthritis and Cartilage. 2006;14(3):279285. doi:10.1016/j.joca.2005.10.002

29. Tejedor A, León F, Lora D, Pérez Á, Vargas F, Gómez A.ArtroPro Study Group. Can an intervention on clinical inertia have an impact on the perception of pain, functionality and quality of life in patients with hip and/or knee osteoarthritis? Results from a cluster randomized trial. Aten Primaria.2012;44(2):65-72. doi:10.1016/j.aprim.2011.01.007 